\title{
Lena Kreck
}

\section{Möglichkeiten und Grenzen des rechtlichen Schutzes für Umweltflüchtlinge"}

Die auf Erdbeben und einen Tsunami folgende nukleare Katastrophe in Japan vernichtete ganze Landstriche. Allein dieses Beispiel macht deutlich, wie eng der Zusammenhang von Umweltveränderungen und Migration ist ${ }^{1}$ und wie drängend die daraus resultierenden Fragen sind. Seit nunmehr gut 25 Jahren wird die Debatte über Umweltflüchtlinge ${ }^{2}$ geführt. Es besteht vor allem Uneinigkeit darüber, wie hoch ihre Zahl anzusetzen ist ${ }^{3}$ und wie sie juristisch geschützt werden können.

Dieser Beitrag wird untersuchen, inwiefern Umweltflüchtlingen Schutz aus der Genfer Flüchtlingskonvention (GFK) ${ }^{4}$ bzw. subsidiärer Schutz zu gewähren ist, um abschließend einen Vorschlag für einen adäquateren Umweltflüchtlingsschutz zu unterbreiten.

\section{Umweltflüchtlingsschutz nach der Genfer Flüchtlingskonvention}

Aus flüchtlingsrechtlicher Sicht lautet die erste Frage: Handelt es sich bei Umweltflüchtlingen um Flüchtlinge im Sinne der GFK? Die GFK bindet als Vertrag nicht nur die Mitgliedstaaten. Sie stellt hinsichtlich des refoulement-Verbots in Art. 33 GFK Völkergewohnheitsrecht dar und ist damit die zentrale völkerrechtliche Rechtsquelle zum Schutze der Flüchtlinge. Flüchtling sind nach der Legaldefinition der GFK all diejenigen, die sich aus begründeter Furcht vor Verfolgung wegen ihrer Rasse, Religion, Nationalität, Zugehörigkeit zu einer bestimmten sozialen Gruppe oder wegen ihrer politischen Überzeugung außerhalb ihres Landes befinden. Lässt man den Umstand außen vor, dass eine Vielzahl der

Besonderer Dank gilt Nora Markard für ihre konstruktive Kritik.

1 Vgl. UNHCR, Climate change, natural disasters and human displacement: a UNHCR perspective, 29.10.2008; CARE, CIESIN, UNHCR, UNU-EHS, and The World Bank, In Search of Shelter, Mapping the Effects of Climate Change on Human Migration and Displacement, 2009, abrufbar unter http:// ciesin.columbia.edu/documents/clim-migr-report-june09_final.pdf (Stand 14.2.2011); Keane, The environmental Causes and Consequences of Migration: A Search for the Meaning of „environmental Refugees", 16 Georgetown International Environmental Law Review 2004, S. 209, 221; Myers, Environmental Refugees in a Globally Warmend World, 43 (11) BioScience 1993, S. 752, 758; Biermann, Umweltflüchtlinge. Ursachen und Lösungsansätze, Aus Politik und Zeitgeschichte, B 12/2001, S. 24; Charnley, Environmentally-Displaced People and the Cascade Effect: Lessons from Tanzania, 25 (4) Human Ecology 1997, S. 593, 595.

2 Der Begriff des Umweltflüchtlings wurde 1985 mit dem Bericht zum Umweltprogramm der Vereinten Nationen (UNEP) in die Diskussion eingebracht. IOM, UNHCR sowie viele Autor_innen scheuen sich davor, den Begriff Umweltflüchtling zu verwenden und bevorzugen Begriffe wie Umweltmigration oder environmental displaced persons. Andere befassen sich alleine mit Klimaflüchtlingen. In diesem Beitrag wird der Begriff Umweltflüchtling verwendet, auch wenn bewusst ist, dass Umweltflüchtling missverständlich gelesen werden kann.

3 Die Schätzungen hinsichtlich der Anzahl der zu erwartenden Umweltflüchtlinge schwanken zwischen mehreren Zehntausenden bis hin zu einer halben Milliarde von Fliehenden im Jahr 2050; vgl. Jakobeit/ Methmann, Klimaflüchtlinge. Die verleugnete Katastrophe, Hamburg 2007, S. 1, 2 ff.

4 Abkommen über die Rechtsstellung der Flüchtlinge vom 28.7.1951, BGBl. 1953 II, S. 560, UNTS Bd. 189 S. 137; in der Fassung des Protokolls über die Rechtsstellung der Flüchtlinge vom 31.1.1967, BGB1. 1969 II, S. 1294, UNTS Bd. 606 S. 267. 
Umweltflüchtlinge Binnenflüchtlinge ${ }^{5}$ sind und damit nicht die Voraussetzung der Grenzüberschreitung erfüllen, so bleibt vor allem zu prüfen, inwiefern eine begründete Furcht vor einer Verfolgung vorliegt, welche aus einem der fünf genannten Gründe erfolgt.

\section{Begründete Furcht vor Umweltveränderungen}

Bei der Diskussion um die Umweltflüchtlinge stellt sich die Frage, was überhaupt unter einem Umweltflüchtling zu verstehen ist. Eine Vielzahl von Definitionen wird diskutiert. Eine jüngst im Auftrag des Umweltbundesamtes (UBA) ${ }^{6}$ veröffentlichte Studie beinhaltet als Arbeitsdefinition, dass Umweltflüchtlinge jene „StaatsbürgerInnen und Personen mit gewöhnlichem Aufenthalt [sind], die ihren Heimatort innerhalb ihres Herkunftsstaates oder ihren Herkunftsstaat vorübergehend oder auf Dauer verlassen mussten; der maßgebliche, unmittelbare Grund für das Verlassen sind von Mensch oder Natur herbeigeführte Umweltveränderungen, die das Leben oder den Lebensunterhalt ernsthaft gefährden. "7 An dieser Stelle interessiert vor allem, ob für eine Umweltflüchtlingseigenschaft ein von der UBA-Studie formuliertes „verlassen Müssen“ tatsächlich vorauszusetzen ist. Ist es also für die Umweltflüchtlingseigenschaft notwendig, dass die betreffende Person unfreiwillig ihren Heimatort verlässt? Die Studie des UBA bejaht dies wie viele weitere Autor_innen, ${ }^{8}$ wenn sie argumentiert, dass „von der Definition [des Umweltflüchtlings] lediglich unfreiwillige Migration umfasst sein soll“. 9

Dieses Vorgehen ist kritisch zu bewerten. Denn wer sich des Kriteriums der Freiwilligkeit/Unfreiwilligkeit bedient, verkennt die Verflechtungen zwischen sozialen und politischen Unterdrückungsmechanismen ${ }^{10}$ und gerät in das Fahrwasser der zu kurz kommenden Zuschreibungen: „freiwillige ,Entscheidung’ Migration - nicht schützenswert“ vs. „unfreiwillige ,Entscheidung' - Flucht schützenswert“ ${ }^{11}$ Die unterstellte Freiwilligkeit der Migration ist ein Mittel, mit dem die Verpflichtungen zum Schutz durch die Staatengemeinschaft in (nationalen) Grenzen gehalten werden soll, insbesondere bei so genannten Wirtschaftsflüchtlingen. Und auch die Umweltflüchtlinge laufen Gefahr, mit dieser Strategie generell aus dem Schutzbereich herausdefiniert zu werden, zumal der Klimawandel häufig die Vernichtung ihrer Existenzgrundlage zur Folge haben und damit auch die wirtschaftlichen Existenzbedingungen tangieren kann.

Die GFK allerdings sagt über Zwang oder Freiwilligkeit nichts aus; erforderlich ist allein eine begründete Furcht vor Verfolgung. Statt die Schutzwürdigkeit der Umweltflüchtlinge an die „Kontrolle“12 der betroffenen Personen über ihre Lebenssituation zu knüpfen, sind deshalb vielmehr die Tatbestandsmerkmale der

5 Vgl. hierzu den Veranstaltungsbericht der Deutschen Gesellschaft für die Vereinten Nationen e.V. vom 4.6.2009, abrufbar unter http://www.dgvn.de/index.php?id=news\&no_cache=1\&tx_ttnews[tt_ news] 2228 tx_ttnews[backPid] $=2228 \mathrm{cHash}=12 \mathrm{e} 00 \mathrm{f} 3174$ (Stand 14.2.2011); zu Binnenflüchtlingen im Generellen: UNHCR, Handbook for the Protection of Internally Displaced Persons, abrufbar unter http://www.unhcr.org/4c2355229.html (Stand 14.2.2011).

6 Ammer/Nowak/Stadlmayr/Hafner, Rechtsstellung und rechtliche Behandlung von Umweltflüchtlingen, Dessau-Roßlau 2010. Die Studie ist von besonderem Interesse, weil sie die bisher größte deutschsprachige Untersuchung zum Themenkomplex der Umweltflüchtlinge darstellt und von einer Bundesbehörde herausgegeben worden ist.

7 Ebd., S. 27.

8 Bates, Environmental Refugees? Classifying Human Migration Caused by Environmental Change, 23 (5) Population and Environment 2002, S. 465, 467.

9 Ammer/Nowak/Stadlmayr/Hafner (Fn. 6), S. 24.

10 Marx, Eine menschenrechtliche Begründung des Asylrechts, Rechtstheoretische und rechtsdogmatische Untersuchung zum Politikbegriff im Asylrecht, Baden-Baden 1984, S. 55 ff.

11 Foster, International refugee law and socio-economic rights: Refuge from deprivation, Cambridge 2007, S. 2 .

12 Ammer/Nowak/Stadlmayr/Hafner (Fn. 6), S. 24 
GFK zu prüfen und zunächst zu fragen, ob bei Umweltveränderungen eine begründete Furcht vor Verfolgung gegeben ist. Von einer begründeten Furcht ist dann zu sprechen, wenn die tatsächliche Gefahr einer Verfolgung besteht. ${ }^{13}$ Dass Umweltveränderungen eine tatsächliche Gefahr darstellen können, ist durchaus denkbar, wie bereits das Beispiel Japans verdeutlicht. Dass diese massiven Umweltveränderungen begründete Furcht verursachen können, ist logische Folge. Soweit auch ein subjektives Element des Sich-Fürchtens für erforderlich gehalten wird, kann das Verlassen des Landes als Indiz gelten.

\section{Umweltveränderungen als Verfolgung}

Voraussetzung für den Schutz der GFK ist es weiterhin, dass die Gefahr für die Umweltflüchtlinge von einer Verfolgungssituation ausgeht. Bei der rechtlichen Konkretisierung des Begriffs der Verfolgung zeigt sich die Verwobenheit von Menschenrechten und Flüchtlingsrecht. So ist die Verletzung grundlegender Menschenrechte flüchtlingsrechtlich als Verfolgung zu fassen, wenn sie eine gewisse Intensitätsschwelle erreicht. ${ }^{14}$ Hiervon sind nicht nur staatliche, sondern auch nichtstaatliche Menschenrechtsverletzungen erfasst, wenn der Staat entweder nicht willens oder nicht in der Lage ist, gegen sie Schutz zu bieten. ${ }^{15}$ Der Schutz der GFK ist ein subsidiärer Menschenrechtsschutz: Versagt der Schutz im Heimatstaat, muss der Aufnahmestaat einspringen. ${ }^{16} \mathrm{Im}$ Falle von weitreichenden Umweltveränderungen, welche ganze Regionen vernichten, ist nicht nur das Menschenrecht auf Leben ${ }^{17}$ gefährdet. Auch das Recht auf Nahrung wie auf Unterkunft ${ }^{18}$ kann häufig nicht gewährleistet werden.

Daran anknüpfend hat Jessica Cooper bereits vor über zehn Jahren die These vertreten, dass eine Verfolgung auch dann gegeben sei, wenn Staaten fluchtverursachende Umweltveränderungen nicht verhindern. ${ }^{19}$ An drei Beispielen zeigt sie, dass sich Menschen durch Umweltveränderungen einer Verfolgung ausgesetzt sehen können. Erstens wirft sie den Regierungen der Sahelzone vor, dass sie es unterlassen hätten, Maßnahmen zu ergreifen, welche die Wüstenbildung hätten eindämmen können. ${ }^{20}$ Dass die Staatengemeinschaft es nicht vermag, sich verbindlich auf eine tatsächliche Reduzierung der Emissionen zu einigen, um den Klimawandel abzuwenden, führt Cooper als weiteres Beispiel für Verfolgung bei Umweltveränderungen an. ${ }^{21}$ Als dritten Punkt nennt sie die Katastrophe von Tschernobyl, die durch die Fixierung der sowjetischen Regierung auf Atomkraft erst entstanden und das durch sie erzeugte Leid durch die verzögerte Evakuierung noch verschlimmert worden sei. ${ }^{22}$ Mit Cooper könnte man im Fall umweltbedingter Flucht daher regelmäßig sowohl staatliche Verfolgung rechtlich begründen als auch nichtstaatliche Verfolgung durch umweltschädigende Un-

Hathaway/Hicks, Is there a Subjective Element in the Refugee Convention's Requirement of „wellfounded Fear"?, 26 Michigan Journal of International Law 2005, S. 505, 507.

14 Hathaway, The Law of Refugee Status, Markham 1991, S. 108.

15 Ebd., S. 127.

16 Ebd., S. 124.

17 Art. 3 Allgemeine Erklärung der Menschenrechte vom 10.12.1948, UNYB (1948-49) S. 535; Art. 6 Abs. 1 Pakt über bürgerliche und politische Rechte vom 19.12.1966, BGBl. 1973 II, S. 1534, UNTS Bd. 999 S. 171.

18 Art. 11 Abs. 1 Pakt über wirtschaftliche, soziale und kulturelle Rechte vom 19.12.1966, BGBl. 1973 II, 1569, UNTS Bd. 993 S. 3.

19 Cooper, Environmental refugees: Meeting the requirements of the refugee definition, 6 N.Y.U. Environmental Law Journal 1998, S. 480, $502 \mathrm{ff}$.

20 Ebd., S. 506.

21 Ebd., S. 513.

22 Ebd., S. $515 \mathrm{f}$. 
ternehmen, gegen die die Staaten keinen Schutz bieten, zum Ausgang der Begründung von GFK-Rechten nehmen.

Gegen diese Lesart von „Verfolgung“ in der Flüchtlingsdefinition der GFK gibt es Widerstand..$^{23}$ So wird argumentiert, dass konkrete Handlungen, deren Folgen bestimmte Personen aus konkreten Gründen träfen, ${ }^{24}$ sowie intentionale Umweltveränderungen ${ }^{25}$ Voraussetzungen für Verfolgungen seien. Hiergegen ist einzuwenden, dass eine dolose Intention, also eine Zielgerichtetheit der Verfolgung im Rahmen der GFK, überwiegend für nicht erforderlich gehalten wird. ${ }^{26}$ Zudem aber würden die Umweltzerstörungen, die zu Umweltflucht führen, mehrheitlich durch Staaten des Nordens, nicht durch die zumeist betroffenen Staaten selbst verursacht. Eine staatliche Verfolgung käme demnach nur durch einen fremden Staat in Betracht, was von der GFK nicht umfasst sei. ${ }^{27}$

Die Schwierigkeit, Umweltflüchtlinge als Verfolgte im Sinne der GFK zu verstehen, liegt jedoch noch auf einer anderen Ebene. Coopers Argumentation ist nämlich grundsätzlich überzeugend: In Zeiten des anthropogenen Klimawandels und unvorstellbarer Industrieunfälle fallen Umweltveränderungen „nicht vom Himmel“, sondern sind auf umweltpolitische und damit menschliche Entscheidungen zurückzuführen. Allein die fehlende Bereitschaft jenseits von Lippenbekenntnissen, die $\mathrm{CO}_{2}$-Belastung unmittelbar und radikal zu reduzieren, sowie die Freigabe risikoreicher Technologien rechtfertigen es, den Staaten grundsätzlich Verantwortung zuzuweisen. Zumeist aber ist kein bestimmbares menschliches Verhalten auszumachen, welches unmittelbar zu einer Menschenrechtsverletzung führt. Die Kausalkette wird nur in Ausnahmefällen direkt zu einem konkreten Akteur führen, etwa zu einem Unternehmen, das Gift in einen Fluss einleitet. In der Regel sind nur indirekte Auswirkungen auf die Umwelt zu verzeichnen, ein unmittelbarer Zusammenhang zwischen menschlicher Handlung und Menschrechtsverletzung kann dann nicht festgestellt werden. Eine völlig akteurlose Verfolgung würde aber den Rahmen der GFK sprengen, so dass nur in wenigen Fällen der Verfolgungsbegriff erfüllt sein wird.

\section{Anknüpfung an einen Konventionsgrund: Zugebörigkeit zu einer bestimmten sozialen Gruppe}

Eine weitere Schwierigkeit bei der Anerkennung von Umweltflüchtlingen als GFK-Flüchtlinge betrifft die Anknüpfung an einen Konventionsgrund. Von der GFK erfasst sind nur diskriminierende Menschenrechtsverletzungen, also solche, die an „Rasse, Religion, Nationalität, Zugehörigkeit zu einer bestimmten sozialen Gruppe oder politische Überzeugung “ anknüpfen. Umweltkatastrophen, so die Annahme, betreffen aber alle Menschen ohne Unterschied. ${ }^{28}$

23 Kolmannskog/Myrstad, 11 European Journal of Migration and Law 2009, S. 313, 314; Lonergan, The Role of Environmental Degradation in Population Displacement, 4 Environmental Change and Security Report 1998, S. 5, 8; Lopez, The Protection of Environmentally-Displaced Persons in International Law, 37 Environmental Law 2007, S.365, 377 ff.; Moberg, Extending Refugee Definitions to Cover Environmentally Displaced Persons Displaces Necessary Protection, 94 Iowa Law Review 2009, S. 1007 , 1113; Zerger, Klima- und umweltbedingte Migration, ZAR 2009, S. 85, 88.

24 Zartner Falstrom, Stemming the flow of environmental displacement: Creating a Convention to Protect Persons and Preserve the Environment, 6 Colorado Journal of International Environmental Law \& Policy 2001, S. 1, 14.

25 King, Environmental Displacement: Coordinating Efforts to find Solutions, 18 Georgetown International Environmental Law Review 2006, S. 543, 553.

26 Hathaway (Fn. 14), S. 104-105.

27 Grote, Der rechtliche Schutz ökologisch motivierter Flucht, abrufbar unter http://netzwerk-migrationsrecht.akademie-rs.de/wp-content/uploads/2009/02/grote-der-rechtliche-schutz-okologisch-motivierter-flucht.pdf (Stand 14.2.2011), S. 7.

28 UNHCR, Handbuch über Verfahren und Kriterien zur Feststellung der Flüchtlingseigenschaft, Genf 1979, Rn. 39. 
Allerdings belegen die Ergebnisse der Vulnerabilitätsforschung in der Stadtsoziologie, welche vor allem im Nachgang des Hurrikans Katrina die spezifischen Auswirkungen von Umweltkatastrophen auf unterschiedliche soziale Gruppen auswertet, dass durchaus bestimmte Gruppen einer besonderen Betroffenheit unterliegen können. So werden gesellschaftliche Minderheiten überdurchschnittlich häufig und heftig von Umweltveränderungen betroffen. ${ }^{29}$ Die Ereignisse in New Orleans haben etwa gezeigt, welche Rolle soziale Schicht, Geschlecht und die ethnische Zugehörigkeit (class, gender, race) hinsichtlich der Verletzlichkeit durch Umweltkatastrophen in der US-amerikanischen Gesellschaft einnehmen: ${ }^{30}$ Bestimmte Personengruppen sind aufgrund von Armut, ${ }^{31}$ persönlichen Abhängigkeiten ${ }^{32}$ und fehlendem Verständnis und Eingehen auf Bedürfnisse von Menschen jenseits der „Mehrheitsgesellschaft“ ${ }^{\text {33 }}$ besonders heftig von Umweltkatastrophen betroffen. Zudem ist festzustellen, dass Aufbauprogramme nach Umweltkatastrophen soziale Ungleichheiten eher verschärfen als mindern, indem Stadtteile, die hauptsächlich von Minderheiten bewohnt werden, nachrangig beim Wiederaufbau unterstützt werden. ${ }^{34}$ Nicht nur die Umweltschäden selbst, sondern auch die Vorenthaltung von Wiederaufbauleistungen - als Verletzungen des Sozialpakts - können daher diskriminierende Verfolgung darstellen.

Tatsächlich gibt es daher (begrenzte) Fälle, in denen Umweltflüchtlinge aufgrund einer Verfolgung wegen der Zugehörigkeit zu einer bestimmten sozialen Gruppe Flüchtlinge nach der GFK sind. Hierfür ist es erforderlich, dass eine unabhängig von der Verfolgung als solche bestehende soziale Gruppe besondere Nachteile erfährt. ${ }^{35}$ Dies ist etwa der Fall, wenn der indische Staat nach dem Tsunami 2004 den Angehörigen der Dalit, der Kaste der „Unberührbaren“, weniger als den Angehörigen anderer Kasten oder gar keine Hilfe gewährt. ${ }^{36}$ In dieser speziellen Konstellation liegt nicht nur eine Verfolgung durch die fehlende staatliche Unterstützung, sondern auch die Anknüpfung an einen Konventionsgrund wegen der diskriminierenden Behandlung einer bestimmten sozialen Gruppe vor.

\section{Subsidiärer Umweltflüchtlingsschutz}

Aber auch wenn nur in begrenzten Fällen Umweltflüchtlinge unter die Definition des Flüchtlings nach der GFK subsumiert werden können, so bedeutet dies nicht, dass sie in allen anderen Fällen zwingend schutzlos sind. Immerhin ist ein subsidiärer Schutz von Umweltflüchtlingen, vor allem nach Art. 3 bzw. nach Art. 8 der Europäischen Menschenrechtskonvention (EMRK), denkbar. ${ }^{37}$ Zwar hat der Europäische Gerichtshof für Menschenrechte (EGMR) bisher keinen Fall

Vgl. Powers, A Matter of Choice, Historical Lessons for Disaster Recovery, in Hartman/Squires (Hrsg.), There is no such Thing as an Natural Disaster, Race, Class, and Hurrica Katrina, Philadelphia 2006, S. 13-33.

30 Tierney, Social Inequality, Hazards, and Disasters, in: Daniels/Kettl/Kunreuther (Hrsg.), On Risk and Disaster, Lessons from Hurricane Katrina, Philadelphia 2006, S. 109-128.

31 Ebd., S. $113 \mathrm{ff}$.

32 Ebd., S. 120.

33 Ebd., S. $117 \mathrm{ff}$.

34 Ebd., S. 123.

35 Vgl. Grote (Fn. 27), S. 6; Löhr, Die kinderspezifische Auslegung des völkerrechtlichen Flüchtlingsbegriffs, Baden-Baden 2009, S. 135.

36 Pelzer, Umweltflüchtlinge ohne Schutz, Eine Ergänzung zum internationalen Flüchtlingsrecht ist notwendig, umwelt aktuell, Dez. 2008/Jan. 2009, S. 29-30.

37 Vgl. Grote (Fn. 27), S. 6; Lohr (Fn. 35), S. 135. 
von Umweltflucht entschieden. Eine Reihe von Entscheidungen ${ }^{38} \mathrm{zu}$ Art. 3

EMRK erlaubt es jedoch dann von einem Abschiebeverbot auszugehen, wenn aufgrund von Umweltveränderungen die Lebensgrundlage zerstört und damit die Betroffenen mit der Abschiebung eine unmenschliche und erniedrigende Behandlung erwartet. Dabei kann eine allgemeine Gefahr durchaus zu einem individuellen Risiko führen, das nicht individualisiert sein muss. ${ }^{39}$ Sehr große Ausnahmen des Schutzes dürften allerdings diejenigen Fälle darstellen, in denen bei Abschiebung eine unmenschliche und erniedrigende Behandlung aufgrund von Gesundheitsgefahren ${ }^{40}$ oder sozialer Not ${ }^{41}$ droht. $^{42}$

Weiter ist ein Abschiebehindernis nach Art. 8 Abs. 1 EMRK wegen eines umweltbedingten Eingriffs in die Privatsphäre zumindest nicht ausgeschlossen. Zwar gewährt die EMRK nach der Rechtsprechung des EGRM keinen Anspruch auf die Herstellung einer sauberen Umwelt, den Betroffenen darf jedoch eine menschenwürdige Alternative zu ihrer belastenden Lebenssituation nicht verwehrt werden. ${ }^{43}$ Dementsprechend ist eine Abschiebung in eine durch Umweltveränderungen unbewohnbare Gegend nicht mit der EMRK in Einklang zu bringen.

Auch die Qualifikationsrichtlinie ${ }^{44}$ der Europäischen Union verpflichtet die Mitgliedstaaten nur bedingt, Umweltflüchtlingen Schutz zu gewähren. Art. 15 (a) und (b) der Richtlinie sind als Abschiebeverbot aufgrund von Umweltveränderungen zu verstehen, wenn massive Umweltveränderungen das Recht auf Leben zu gefährden drohen oder die Voraussetzungen des Art. 3 EMRK erfüllt sind. ${ }^{45}$ Ähnlich verhält es sich bei der Richtlinie über Mindestnormen für die Gewährung vorübergehenden Schutzes im Falle eines Massenzustroms von Vertriebenen, ${ }^{46}$ also von Personen, „die ihr Herkunftsland oder ihre Herkunftsregion haben verlassen müssen oder insbesondere nach einem entsprechenden Aufruf internationaler Organisationen evakuiert wurden und wegen der in diesem Land herrschenden Lage nicht sicher und dauerhaft zurückkehren können“. Hier ist der Schutz für „Umweltflüchtlinge“ aufgrund der temporären Schutzgewährung von maximal drei Jahren stark eingeschränkt, was umso schwerer wiegt, weil die Schutzgewährung im Ermessen der Mitgliedstaaten liegt, welche zu einer gemeinsamen Entscheidung kommen müssen. ${ }^{47}$ Diese Richtlinie gewährt also kein unmittelbares individuelles Recht.

ECHR, Selcuk and Asker v. Turkey, 24.4.1998, Appl. No. 12/1997/796/998-999; ECHR, Bilgin v. Turkey, 16.11.2000, Appl. No. 23819/94; ECHR, Dulas v. Turkey, 30.1.2001, Appl. No. 25801/94; ECHR, Bensaid v. UK, 6.5.2001, Appl. No. 44599/98; ECHR, H.L.R. v. France, 22.4.1997, Appl. No. 11/1996/630/813; ECHR, D. v. UK, 2.5.1997, Appl. No. 146/1996/767/964.

39 ECHR, NA. v. UK, 17.7.2008, Appl. No. 25904/07.

40 ECHR, D. v. UK, 2.5.1997, Appl. No. 146/1996/767/964.

41 ECHR, Z and others v. UK, 10.5.2001, Appl. No. 29392/95.

42 Ammer, Climate change and Human Rights: The Status of Climate Refugees in Europe, Swiss Initiative to Commemorate the 60th Anniversary of the UDHR, 2009, S. 60.

43 ECHR, X and Y v. Germany, 13.5.1976, Appl. No. 7407/76; ECHR, G and E v. Norway, 3.10.1983, Appl. No. 9278/81, 9415/81; ECHR, Arrondelle v. UK, 15.7.1980, Appl. No. 7889/77 (durch gütliche Einigung beigelegt); ECHR, Baggs v. UK, 16.10.1985, Appl. No. 9310/81 (durch gütliche Einigung beigelegt); ECHR, Powell and Rayner v. UK, 21.2.1990, Appl. No. 9310/81; ECHR, Lopéz Ostra v. Spain, 9.12.1994, Appl. No. 16798/90.

44 Richtlinie 2004/83/EG vom 29.4.2004 über Mindestnormen für die Anerkennung und den Status von Drittstaatsangehörigen oder Staatenlosen als Flüchtlinge oder als Personen, die anderweitig internationalen Schutz benötigen, und über den Inhalt des zu gewährenden Schutzes, ABl. EG L 304, S. 12.

45 Kolmannskog/Myrstad (Fn. 23). S. 322.

46 Richtlinie 2001/55/EG vom 20.07.2001 über Mindestnormen für die Gewährung vorübergehenden Schutzes im Falle eines Massenzustroms von Vertriebenen und Maßnahmen zur Förderung einer ausgewogenen Verteilung der Belastungen, die mit der Aufnahme dieser Personen und den Folgen dieser Aufnahme verbunden sind, auf die Mitgliedstaaten, ABl. EG L 212, S. 12.

47 Kolmannskog/Myrstad (Fn. 23), S. 318. 
Wenn man also zu dem Schluss kommt, dass nur unter sehr besonderen und beschränkten Voraussetzungen Umweltflüchtlinge nach geltendem Recht als schutzwürdig verstanden werden, so drängt sich die Frage nach wirkungsvollen Instrumenten zum Schutze der Umweltflüchtlinge auf. Denn wenn Umweltveränderungen das Leben oder die Gesundheit von Menschen in gleicher Weise bedrohen wie gezielte Verfolgungshandlungen, erscheint ein flüchtlingsrechtliches Schulterzucken unbefriedigend. Dementsprechend werden unterschiedliche Ansätze zum Umweltflüchtlingsschutz diskutiert: Sie reichen von einer Umweltflüchtlingskonvention, welche an die Antifolterkonvention ${ }^{48}$ angelehnt ist, ${ }^{49}$ bis hin zur Ergänzung der Klimarahmenkonvention ${ }^{50}$ um ein Zusatzprotokoll zum Schutze der Klimaflüchtlinge. ${ }^{51}$

Besser erscheint es jedoch, sich bei der Schaffung eines neuen völkerrechtlichen Instruments an den Voraussetzungen der GFK für eine Flüchtlingseigenschaft zu orientieren. Dies bietet sich an, da Umweltflüchtlinge zwar nur in sehr beschränken Konstellationen als Verfolgte im Sinne der GFK verstanden werden können, doch eine immerhin „mittelbare Verfolgung“ durch Umweltveränderungen nicht von der Hand zu weisen ist. Es ist somit naheliegend, die Normierung eines Umweltflüchtlingsschutzes deutlich an der Flüchtlingsdefinition der GFK zu orientieren. Dies würde auch zu einer Aufwertung der Umweltflüchtlinge führen, die dann zwar nur komplementär Schutz erfahren könnten. Eine gewisse Parallele zwischen Umweltflüchtlingen und Konventionsflüchtlingen könnte sichtbar gemachen werden. Allerdings müsste hierfür nicht nur der Verfolgungsbegriff modifiziert, sondern auch auf das Erfordernis einer diskriminierenden Behandlung verzichtet werden. Im Kern geht es deshalb darum, für diejenigen ein Aufenthaltsrecht zu normieren, welche in ihrem Herkunftsland eine Bedrohung aufgrund von Umweltveränderungen zu fürchten haben. Eine solche Bedrohung könnte beinhalten, dass die persönliche Existenz vernichtet worden ist und die infrastrukturellen sowie finanziellen Voraussetzungen nicht genügen, um in kurzer Zeit ein sicheres und gesundes Leben führen zu können.

Da eine Großzahl der von Umweltveränderungen Betroffenen ihr Heimatland nicht verlassen, wären die Mitgliedstaaten neben dem subsidiären Flüchtlingsschutz zu weiteren Maßnahmen zu verpflichten. Hierzu könnten Entschädigungszahlungen zum Wiederaufbau (sofern möglich) aus einem Umweltflüchtlingsfonds zählen, wenn kein unmittelbarer Verursacher auszumachen ist. Doch all dies darf darüber nicht hinwegtäuschen, dass ein umfassendes Umweltflüchtlingsrecht nur die Folgen, nicht aber die Ursachen bekämpfen könnte.

50 Rahmenkonvention der Vereinten Nationen über Klimaänderungen vom 9.5.1992, BGBl 1993 II, S. 1784; ILM 31 (1992) S. 851.

51 Biermann/Boas, Für ein Protokoll zum Schutz von Klimaflüchtlingen - Global Governance zur Anpassung an eine wärmere Welt, 1 Vereinte Nationen 2008, S. 10, $11 \mathrm{ff}$. 